\section{LETTERS \\ Do I need to be an endocrinologist to diagnose Cushing's disease?}

Around October 1999, while playing cricket after a long time, I fell down for no apparen reason. The same event happened a month later. Being mainly concerned about unexplained falls, I sought help from neurological colleagues. Two neurologists examined me and both reassured me: "your exam is normal and there is nothing to warrant any further investigations". I checked my vitamin $\mathrm{B} 12$, which was $81.0 \mathrm{pg} / \mathrm{ml}$ (normal range 200-900). A combination of B12 deficiency and proximal myopathy could explain my falls. Having put on $4 \mathrm{~kg}$ over the past year, I sought advice from an internist. More concerning was that the central weight gain was starting to affect my appearance. The internist ascribed it to middle age spread and having checked my glucose and lipids, referred me to our nutritionist. The nutritionist having seen the results gave a good diet and exercise plan. Following this diet and exercise plan, I developed pain in my left foot and it got so severe that I presented to our radiologist with an $x$-ray film. On first look, he dismissed it as normal but on closer look identified a hairline fracture in the fifth metatarsal. I was referred to an orthopaedic surgeon who advised me to avoid brisk walks for two weeks.

I questioned both of them-why did I break my bone? I was told that it may happen with strenuous workouts. Now I started to notice a change in my sleep, mood, and thoughts. I began to wake up about 3-4 am for no good reason and it was difficult to go back to sleep. Though unable to have a good night's sleep of seven hours, which used to be a "must" for me I was still full of energy during the day but would develop a headache at the end of a busy clinic. I sought advice from my psychiatric colleague. "It appears like mania or hypomania but why should you have it? Forget it, or if you like you may try some sedatives". For headache, no recommendation.

I asked my staff nurse to check my blood pressure in the busy clinic and it was 180/100 $\mathrm{mm} \mathrm{Hg}$. Not surprisingly, I got more and more worried and one day while doing an endocrine clinic, decided to investigate myself.

While asking for routine analyses, I decided to include thyroid function tests and also cortisol. My cortisol (1600 hours) was $588 \mathrm{nmol} /$ (140-690), then looking back over the past months, it occurred to me that all of these fea tures could be explained by one syndrome, Cushing's, but for the low B12. I submitted sample for 24 hour urine cortisol and reviewed the literature for B12 and cortisol. The 24 hour urine cortisol was $1644 \mathrm{nmol} /$ day (110-436).

I emailed the case to my mentors, Professors A B Grossman and R J M Ross, also mentioning that I had found reports of B 12 deficiency producing circadian rhythm abnormalities and dexamethasone non-suppression. ${ }^{1}$ I was advised to replace $\mathrm{B} 12$ and then repeat the tests. The repeat 24 hour urine cortisol was 466 $\mathrm{nmol} /$ day and overnight dexamethasone suppression test $66 \mathrm{nmol} / \mathrm{l}$. However, after completing an empiric six injection course over on month, I repeated the same tests and the 24 hour urine low dose cortisol was $1911 \mathrm{nmol} /$ day (110-436) and low dose dexamethasone suppression $295 \mathrm{nmol} / \mathrm{l}(<50)$.

Subsequent investigations and imaging showed pituitary dependent Cushing's disease. I underwent transsphenoidal selective adenomectomy by a neurosurgeon colleague in my own centre. Postoperatively, I had undetectable cortisol and normal pituitary function.

This case illustrates the complexity involved in the diagnosis of Cushing's disease. It also illustrates two points that physicians should be aware of when consulted by other physicians: "treat physicians as patients when they come for advice for medical problems" and "physicians could also develop any illness and should be diagnosed". The question about B12 deficiency remains unanswered.

A Jabbar

Diabetes and Endocrine Section, The Aga Khan University, Pakistan; abdul.jabbar@akunet.org

\section{Reference}

1 James SP, Golden RN, Sack DA. Vitamin B 12 deficiency and the dexamethasone suppression

\section{Thyrotoxic periodic paralysis in western countries}

I read with interest the case report by Dixon and Jones on thyrotoxic periodic paralysis (TPP) in a white woman. ${ }^{1}$ TPP is, indeed, extremely rare in women and also in the white population. However, TPP is an extremely common presentation with hypokalaemia in Orientals. ${ }^{2}$ It is imperative that any person of Oriental origin presenting with hypokalaemic weakness has their thyroid status checked ${ }^{2}$. doctors are quite likely to face this situation in the west because of increased immigration.

Attacks of TPP are very common in the early hours of the night, ${ }^{3}$ but unfortunately there was no mention of the time of occurrence in the case reported.

The authors confirmed that their patient did not require $\beta$-blockers, yet this is standard practice in this condition as potassium replacement in this sort of hypokalaemia can cause rebound hyperkalaemia. ${ }^{4}$ I agree that the signs and symptoms of thyrotoxicosis in TPP may be subtle in the west, but this is in contradiction to the Asian experience.

It is, indeed, puzzling as to why there is a male predominance in TPP with hypokalaemia, while Graves' disease is more common in females. It is, however, more puzzling as to why there is a racial difference in this condition. I agree with the authors that there may be a difference in the HLA subtypes. HLA subtypes in different ethnic groups with TPP have been widely studied. HLA DRw8 makes the Japanese more susceptible to TPP, and yet the same HLA subtype in the white population make them susceptible to Graves' disease, but without periodic paralysis. ${ }^{5}$ This phenomenon contradicts the authors' view that there may be a difference in the HLA subtypes and whether one would develop TPP or not, with the background of Graves' disease. Within the same HLA subtype, a genetic mutation in the control of sodium-potassium ATPase activity or some other environmental factor may be the explanation.

I certainly cannot agree with the view that once these patients are euthyroid that they are not at increased risk of hypokalaemic paralysis Control of Graves' disease with antithyroid drugs is very variable. Altogether $70 \%$ may relapse during the course of treatment, and therefore are at risk of developing TPP. Therefore, it has been suggested that $\mathrm{I}^{131}$ ablation in TPP may need to be carried out, irrespective of age at presentation, because of the dangers of severe hypokalaemia. However, the caveat is that even in this group of ablated patients there is a risk of paralytic episodes, even with thyroxine replacement, which invariably this group will require in the longer term.

R Sinharay

Tameside General Hospital, Fountain Street, Ashton-Under-Lyne, Lancashire OL6 9RW, UK

\section{References} ranjitsinharay@hotmail.com

Dixon AN, Jones R. Thyrotoxic periodic paralysis in a white woman. Postgrad Med J 8.687-8.

2 Ko GTC, Chow CC, Yeung VTF, et al. Thyrotoxic periodic paralysis in a Chinese population. Q J Med 1996;89:463-8.

3 Charness ME, Johns RJ. Hypokalaemic paralysis. Johns Hopkins Med

4 Lin YF, Lin SH, Tsai WS, et al. Severe hypokalaemia in a Chinese male. Q J Med 2002;95:695-704

Lazarus JH. Hyperthyroidism. Lancet 1997;349:339-43.

\section{Authors' reply}

We welcome the comments made by Sinharay. They certainly highlight some of the controversies surrounding this condition that we raised in the case presented. We certainly agree that all patients presenting with hypokalaemic paralysis should have their thyroid status checked as, especially in white patients, signs and symptoms of thyrotoxicosis may be subtle and therefore we hightlighted this is the summary points.

This patient did in fact present in the evening. We were not involved in the patient's initial management, but we find it hard to criticise the initial treatment by on-call doctors to use oral and intravenous potassium supplements as the diagnose of thyrotoxicosis had not been made (and was not suspected) and thyroid function tests (TFTs) are not available out-of-hours. Serum potassium levels were closely monitored. The results from TFTs were only received two days later when the patient was normokalaemic and off potassium supplements. We do agree that there is a risk of hyperkalaemia by using this potassium supplements and therefore highlighted this in our discussion.

We welcome Sinharay's comments on the puzzling male predominance of TPP in a condition that has a female preponderance and that any explanations at present are speculative.

Sinharay disagrees with our comment that when patients are euthyroid they are not at risk of hypokalaemic paralysis. This is contrary to previous experience. ${ }^{12}$ However, we do agree that patients are at risk of hypokalaemic paralysis if thyrotoxicosis reoccurs. As there is a risk of relapse of hypokalaemic paralysis with recurrence of thyrotoxicosis we agree that there is a case to be made for early definitive treatment.

\section{References}

McFadzean AJS, Yeung R. Periodic paralysi complicating thyrotoxicosis in Chinese. BMU $1: 451-5$

Ahlawat SK Sachdev A. Hypokalaemic paralysis. Postgrad Med J 1999;75:193-7. 